\title{
Generalized Software Reliability Model Considering Uncertainty and Dynamics: Model and Applications
}

\author{
Kiyoshi Honda*\&, Hironori Washizaki*,+, and Yoshiaki Fukazawa*,\| \\ ${ }^{*}$ Department of Computer Science and Engineering \\ Waseda University, 3-4-1 Ohkubo \\ Shijuku-ku, 169-8555 Tokyo, Japan \\ ${ }^{\dagger}$ National Institute of Informatics \\ 2-1-2 Hitotsubashi, Chiyoda-ku, 101-8430 Tokyo, Japan \\ SSystem Information Co., Ltd. \\ 1-7-3 Kachidoki, Chuo-ku, 104-0054 Tokyo, Japan \\ \$khonda@ruri.waseda.jp \\ "washizaki.waseda.jp \\ Ifukazawa.waseda.jp
}

Received 23 July 2015

Revised 22 November 2015

Accepted 20 September 2016

\begin{abstract}
Today's development environment has changed drastically; the development periods are shorter than ever and the number of team members has increased. Consequently, controlling the activities and predicting when a development will end are difficult tasks. To adapt to changes, we propose a generalized software reliability model (GSRM) based on a stochastic process to simulate developments, which include uncertainties and dynamics such as unpredictable changes in the requirements and the number of team members. We assess two actual datasets using our formulated equations, which are related to three types of development uncertainties by employing simple approximations in GSRM. The results show that developments can be evaluated quantitatively. Additionally, a comparison of GSRM with existing software reliability models confirms that the approximation by GSRM is more precise than those by existing models.
\end{abstract}

Keywords: Software reliability models; fault prediction; project management.

\section{Introduction}

Software reliability is a critical component of computer system availability. Software reliability growth models (SRGMs), such as the Times Between Failures Model and

$\S$ Corresponding author.

This is an Open Access article published by World Scientific Publishing Company. It is distributed under the terms of the Creative Commons Attribution 4.0 (CC-BY) License. Further distribution of this work is permitted, provided the original work is properly cited. 
Failure Count Model [7], can indicate whether a sufficient number of faults have been removed to release the software. Although the logistic and Gompertz curves [38] are both well-known software reliability growth curves, neither can account for the dynamics of software development because developments are affected by various elements of the development environment (e.g. skills of the development team and changing requirements).

Our research has employed the Failure Count Model, which is based on counting failures and probability methods. Representatives of this type of model include the Goel-Okumoto NHPP Model and the Musa Execution Time Model [7]. Recent studies by Tamura [31], Yamada [37], Zhang [41], Cai [4], Kamei [20], Dohi [5], Schneidewind [27], Nguyen [24] and Okamura [25] have attempted to describe the dynamics of developments using a stochastic process. Many models have been proposed, surveyed, and compared [3, 22, 34]. However, most failure count models cannot account for the dynamics of development (e.g. drastic changes in the development team composition or significant reductions in the development time), and consequently cannot precisely predict when developments will end. Existing methods assume that each parameter is independent of time, which leads to the inability to account for dynamics. We hypothesize that if several developers suddenly join a project in which a SRGM is applied, the development environment suddenly changes, resulting in several influences, especially on the SRGM parameters since such sudden changes should be treated with time-dependent parameters. These assumptions limit the models but also make the models solvable by mathematical methods. For example, the NHPP model has two parameters (i.e. the total number of faults and fault detection rate), which are independent of time because the NHPP model equations cannot be solved if these values have time dependencies.

Previous studies only use linear stochastic differential equations, but our research indicates that nonlinear stochastic differential equations more realistically model actual situations. These studies and SRGMs treat and test several datasets only in the given situation (e.g. within the same company or organization). In short, existing models are tested and applied to the situation from which the datasets are obtained, and are evaluated from different domains. For example, one work evaluated several SRGMs with automotive software datasets, while another assessed two SRGMs with an army system dataset. Rana et al. studied four software projects from the automotive sector and concluded two statistic SRGMs perform better than other SRGMs [26]. On the other hand, Goel et al. investigated two stochastic SRGMs with a U.S. Navy project dataset and concluded their model provides a plausible description [8]. These studies did not evaluate existing SRGMs with other domains.

Herein we propose a model called the Generalized Software Reliability Model (GSRM) [14] to describe several development situations that involve random factors (e.g. team skills and development environment) to estimate the time that a development will end [11]. Additionally, we have predicted the release times of open source software (OSS) using GSRM [15] and agile development [33]. Moreover, we have 
applied GSRM and SRGMs to company's datasets [11-13, 16]. Due to random factors, the GSRM in each situation has an upper and lower limit, suggesting that a GSRM can predict the maximum and minimum number of faults. We formulate the upper and lower limit equations for three development situations with approximations in order to treat these equations easily and predict the number of faults in several ranges. We evaluate and test GSRM and other models using datasets from different organizations and circumstances. GSRM can quantify uncertainties that are influenced by random factors (e.g. team skills and development environments), which is important to more accurately model the growth of software reliability and to optimize development teams or environments.

This study aims to answer the following research questions:

(1) RQ1: Can GSRM be applied to several development situations?

(2) RQ2: Is GSRM an improvement over other models (e.g. NHPP) in describing the growth of software reliability under different situations?

Our contributions are as follows:

(1) We propose a software reliability model applicable to nine development situations, which is $12 \%$ more precise than existing models for recent datasets.

(2) Three approximate equations about three uncertainty types in our software reliability model.

(3) An evaluation with actual datasets confirms that our software reliability model can classify development situations.

To evaluate our model, we simulate nine types of development situations using the Monte Carlo method. To simplify the application of our model, we divide the situations related to uncertainty situations into three types and derive an approximation equation for each. Finally, we apply the approximation equations to four projects from two different organizations and classify these projects into three uncertainty types.

The rest of this paper is organized as follows. Section 2 introduces the non-homogeneous Poisson process (NHPP) SRAM. Section 3 describes our model as a generalized software reliability model and summarizes the types of developments depending on dynamics and uncertainties. In addition, we derive three equations, which depend on the uncertainties. Section 4 applies GSRM to four projects using two datasets and evaluates whether GSRM can accommodate different situations. Moreover, we compare GSRM with NHPP models by focusing on how closely the models can simulate actual data. Section 5 discusses related works, while Sec. 6 provides the conclusion.

\section{Background}

Software reliability is important to release software. Several approaches have been proposed to measure reliability. One is to model faults growth, which is a type of SRGM. Because software development includes numerous uncertainties and 
dynamics regarding development processes and circumstances, this section explains SRGM, its uncertainties and dynamics as well as provides a motivating example.

\subsection{SRGM}

Some models assess software reliability quantitatively from the fault data observed in the testing phase. Similar to these software reliability models, our approach is also based on the fault counting [7] model.

Many software reliability models have been proposed, but the most popular is the non-homogeneous Poisson process (NHPP) model. In this study, NHPP models are compared with GSRM using development data containing the number of faults detected in a given time. Our GSRM is formulated by counting the number of faults detected in a given time assuming that fault detection is based on a stochastic process, whereas the NHPP model assumes that a stochastic process governing the relationship between fault detection and a given time interval is a Poisson process. In actual developments, fault counting predicts the number of remaining faults.

In the NHPP model, the probability of detecting $n$ faults is described by

$$
\operatorname{Pr}\{N(t)=n\}=\frac{\{H(t)\}^{n}}{n !} \exp \{-H(t)\},
$$

where $N(t)$ is the number of faults detected by time $t$ and $H(t)$ is the expected cumulative number of detected faults [35]. Assuming that the number of all faults is constant at $N_{\text {max }}$, the number of detected faults at a unit time is proportional to the number of remaining faults. These assumptions yield the following equation:

$$
\frac{d H(t)}{d t}=c\left(N_{\max }-H(t)\right)
$$

where $c$ is a proportionality constant. The solution of this equation is

$$
H(t)=N_{\max }(1-\exp (-c t)) .
$$

This model is called the exponential SRAM, and was originally proposed by Goel and Okumoto [8]. Yamada et al. derived the delayed S-shaped software reliability growth (S-shaped) model from Eq. (3) with a fault isolation process [39]. Equation (3) can be rewritten into the delayed S-shaped SRGM using $H_{\mathrm{S}}(t)$, which is the expected cumulative number of faults detected in the S-shaped model, as

$$
H_{\mathrm{S}}(t)=N_{\max }\{1-(1+c t) \exp (-c t)\} .
$$

In this paper, we compare GSRM with these two models. Equation (3) results in an exponentially shaped software reliability graph. However, a real software reliability graph typically follows a logistic curve or a Gompertz curve [38], which is more complex. Therefore, we propose a new model that can express either a logistic curve or an exponentially shaped curve for use in actual developments. 


\subsection{Uncertainty and dynamics}

Software development projects have uncertainties and risks. Wallace et al. analyzed the software project risks to reduce the incidence of failure [32]. They mentioned that software projects have six dimensions: Team Risk, Organizational Environment Risk, Requirements Risk, Planning and Control Risk, User Risk, and Complexity Risk. They emphasized that Organizational Environment Risk and Requirements Risk are due to risks and uncertainties. However, existing software reliability growth models do not contain these uncertainty elements. On the other hand, several SRGMs treat limited time-dependent assumptions. Yamada et al. proposed an extended NHPP model related to the test-domain dependence [40]. The test-domain dependent model includes the notion that a tester's skills improve in degrees. Although growth of skills is a time-dependent additional assumption to the NHPP model, this model does not correspond to dynamic changes (e.g. changes in or the number of team members).

\subsection{Motivating example}

Existing studies describe usages and evaluate models, and almost all compare models and try to suggest an improvement. Existing models can be applied to similar situations, but they cannot be applied different situations like other domains or scales. For example, Fig. 1 shows the impact of applying two existing models to different datasets in Refs. 8 and 29, which belong to different organizations. The crosses for "Dataset 1" represent the actual data in [8] and the crosses for "Dataset 2 release 2" represent one project of the actual data in [29]. The dashed lines represent the NHPP model fitted to the actual data. The dotted-dashed lines represent the S-shaped model fitted to the actual data. These results indicate that the fitness of the S-shaped model is greater than the NHPP model in "Dataset 1". However, the fitness

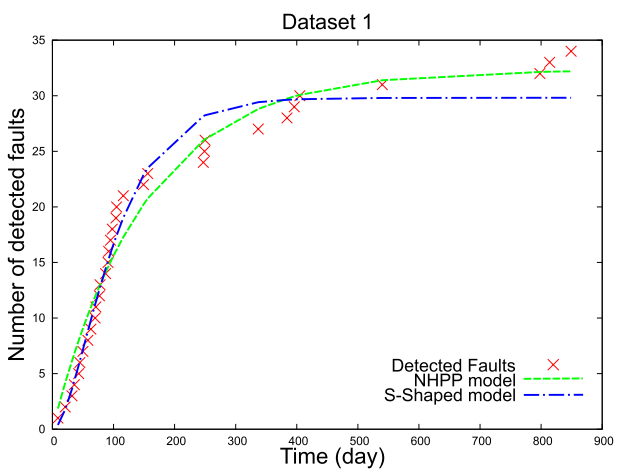

(a)

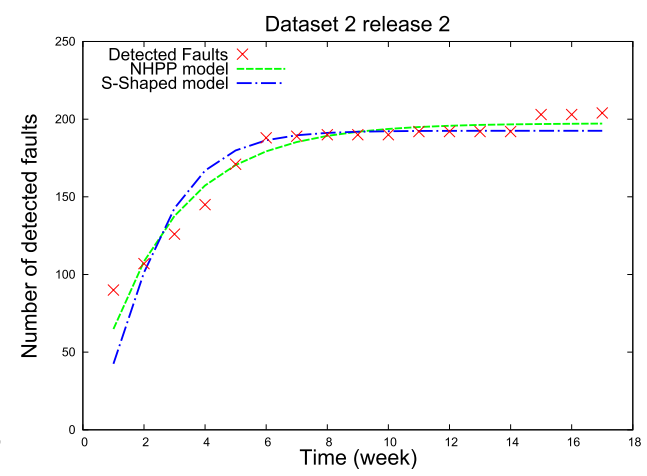

(b)

Fig. 1. Cumulative number of detected faults for Dataset 1 (a) and Dataset 2 release 2 (b) as a function of elapsed time. In the legends, Detected faults, NHPP model, and S-shaped model represent the actual data, the fit using the NHPP model, and the fit using the S-shaped NHPP model, respectively. 
of the NHPP model is greater than the S-shaped model in "Dataset 2 release 2". In other words, the fitness of a model depends on the situation and is not universal. Therefore, we propose a model that is suitable for different situations.

\section{Generalized Software Reliability Model (GSRM)}

For our software reliability model [14], we extend a nonlinear differential equation that describes the fault content as a logistic curve as an $\mathrm{It}_{o}$ type stochastic differential equation. In this paper, we derive three equations corresponding to three types of uncertainties [14]. Moreover, we apply and evaluate the three equations by using actual datasets.

In our previous paper [14], we proposed a simple GSRM equation, which only simulated several development situations that involved random factors. In [14], we could not analyze actual datasets. In this paper, we extend the simple GSRM equation to apply it to actual datasets. The equation is divided into three uncertainty types: late, constant, and early types. Using clearly defined equations, we apply GSRMs to actual datasets and obtain the upper and lower limits in each situation due to random factors, which mean that the GSRM can predict the maximum and minimum number of faults. Additionally, we evaluate and test our GSRMs and other models using datasets from different organizations and circumstances. The GSRM can quantify uncertainties that are influenced by random factors, which is important to more accurately model the growth of software reliability and to optimize development teams or environments.

We start with the logistic differential equation, which is expressed as

$$
\frac{d N(t)}{d t}=N(t)(a+b N(t))
$$

where $N(t)$ is the number of detected faults by time $t, a$ defines the growth rate, and $b$ is the carrying capacity. If $b=0$, then the solutions are exponential functions. Because the numerous uncertainties and dynamic changes prevent actual developments from correctly obeying Eq. (5), it should be extended into a stochastic differential equation. We assume that such dynamic elements are time dependent and contain uncertainties. These elements are expressed using $a$. The time dependence of $a$ can be used to describe situations such as an improved development skills and increased growth rate. The uncertainty of $a$ can describe parameters such as the variability of development members and the environment. The growth of software is analyzed with an emphasis on the testing phase by simulating the number of detected faults. We assume that software development has the following properties:

(1) The total number of faults is constant.

(2) The number of faults that can be found depends on time.

(3) The number of faults that can be found contains uncertainty, which can be simulated with Gaussian white noise. 
The first assumption means that the total number of faults is finite and can be treated as a boundary condition. This assumption implies that correcting faults does not create new ones. Indeed, many SRGMs assume that the total number of faults is constant [22]. Several researchers have proposed SRGMs that can treat infinite faults [23]. We assume that the debugging process creates new faults. We suppose that we can use one model for fault creation and fault detection in the debugging process.

The second assumption means that the ability to detect the faults varies; the ability depends on time because the number of developers changes in recent developments, and the number of developers affects the ability to detect the faults. Researchers have proposed several time-dependent models whose situations are limited such as the error detection per time increases with the progress of software testing [39]. Hou et al. proposed a SRGM upon considering two learning curves (the exponential learning curve and the S-shaped learning curve) [17]. These existing models assume that the number of developers does not change and the time-dependent parameters are a specific model like the exponential learning curve. In contrast, our model does not depend on a specific model.

The third assumption means that uncertainties in the development process affect the ability to detect faults. This assumption is due to the fact that actual datasets have non-constant detection rates or seem to be independent of time. Analyzing actual faults in datasets, we observed several sudden increases in the number of detected faults. Then we modeled the uncertainty, which affects the ability to detect faults as Gaussian white noise that is a simple but commonly used noise. To the best of our knowledge, other SRGMs do not treat such uncertainties that occur in the development. By analyzing the uncertainties for each development, we can understand how a development progresses and predict the progress with a concrete tolerance.

\subsection{Modeling uncertainties and dynamics}

Considering these properties, Eq. (5) can be extended to an $\mathrm{It}_{o}$ type stochastic differential equation with $a(t)=\alpha(t)+\sigma(t) d w(t)$, which is expressed as

$$
d N(t)=(\alpha(t)+\beta N(t)) N(t) d t+N(t) \sigma(t) d w(t)
$$

where $N(t)$ is the number of detected faults by time $t, \alpha(t)+\sigma(t) d w(t)$ is the differential of the number of detected faults per unit time, $\gamma(t)=N(t) \sigma(t) d w(t)$ is the uncertainty term, $\sigma(t)$ is the dispersion, and $\beta$ is the nonlinear carrying capacity term. This equation has two significant terms, $\alpha(t)$ and $\sigma(t) d w(t) ; \alpha(t)$ affects the end point of development and $\sigma(t) d w(t)$ affects the growth curve through uncertainties. Thus, our model treats both uncertainties and dynamics. However, uncertainties cannot be treated directly because they depend on the cause. Our approach treats the uncertainties through a fault detecting process. 


\subsection{Uncertainties}

In particular, Eq. (6) indicates that the stochastic term is dependent on $N(t)$, which means that the uncertainties depend on the number of detected faults. According to Eq. (6), as the number of detected faults increases, the stochastic term has a greater effect on the number of detected faults. Such a situation corresponds to software development with late uncertainty.

We compare three different types of dependencies of $\gamma(t)$ on $N(t)$ :

- The late uncertainty type is where $\gamma(t)=N(t) \sigma d w(t)$.

- The constant uncertainty type is where $\gamma(t)$ is independent of $N(t): \gamma(t)=\sigma d w(t)$.

- The early uncertainty type is where $\gamma(t)$ depends on the inverse of $N(t)$ : $\gamma(t)=1 / N(t) \sigma d w(t)$.

As $\alpha(t)$ and the coefficient of $d w(t)$ are varied, models are simulated using Eq. (6). Table 1 summarizes the types of $\alpha(t)$, the coefficient of $d w(t)$, and the corresponding situations. Using GSRM, the type must be chosen for Table 1 to calculate the parameters using past data. In development, faults are detected and debugged. The detected faults are counted and used to predict when the project will end. Projects contain a lot of uncertainty elements, and the predicted development period is

Table 1. Combinations of dynamics as characterized by $\alpha(t)$ and $\gamma(t) \cdot \alpha(t)$ and $\gamma(t)$ indicate the number of detected faults per unit time and the uncertainty term, respectively.

\begin{tabular}{|c|c|c|c|}
\hline & $\gamma(t)=N(t) \sigma d w(t)$ & $\gamma(t)=\sigma d w(t)$ & $\gamma(t)=\sigma d w(t) / N(t)$ \\
\hline$\alpha_{1}(t)=a_{1}$ (const.) & $\begin{array}{l}\text { The number of detected } \\
\text { faults per unit time is } \\
\text { constant, but the } \\
\text { uncertainty increases } \\
\text { near the end. This } \\
\text { model is similar to a } \\
\text { logistic curve. } \\
\text { (Model 1-1) }\end{array}$ & $\begin{array}{l}\text { The number of detected } \\
\text { faults per unit time } \\
\text { and uncertainty are } \\
\text { constant. (Model 1-2) }\end{array}$ & $\begin{array}{l}\text { The number of detected } \\
\text { faults per unit time is } \\
\text { constant, but the } \\
\text { uncertainty decrea- } \\
\text { ses over time (e.g. } \\
\text { the team matures } \\
\text { over time). (Model } \\
\mathbf{1 - 3})\end{array}$ \\
\hline $\begin{array}{l}\alpha_{2}(t)=a_{2}\left(t<t_{1}\right) \\
\alpha_{2}(t)=a_{3}\left(t \geq t_{1}\right)\end{array}$ & $\begin{array}{l}\text { The number of detected } \\
\text { faults per unit time } \\
\text { changes at } t_{1} \text {, and } \\
\text { the uncertainty } \\
\text { increases near the } \\
\text { end (e.g. new mem- } \\
\text { bers join the project } \\
\text { at time } t_{1} \text { ). (Model } \\
\mathbf{2 - 1})\end{array}$ & $\begin{array}{l}\text { The number of detected } \\
\text { faults per unit time } \\
\text { changes at } t_{1} \text {, but the } \\
\text { uncertainty is con- } \\
\text { stant. (Model 2-2) }\end{array}$ & $\begin{array}{l}\text { The number of detected } \\
\text { faults per unit time } \\
\text { changes at } t_{1} \text {, but } \\
\text { the uncertainty } \\
\text { decreases over time. } \\
\text { (Model 2-3) }\end{array}$ \\
\hline$\alpha_{3}(t) \propto t$ & $\begin{array}{l}\text { The number of detected } \\
\text { faults per unit time } \\
\text { and the uncertainty } \\
\text { increase near the end } \\
\text { (e.g. increasing man- } \\
\text { power with time). } \\
\text { (Model 3-1) }\end{array}$ & $\begin{array}{l}\text { The number of detected } \\
\text { faults per unit time } \\
\text { increases, but the un- } \\
\text { certainty is constant. } \\
\text { (Model 3-2) }\end{array}$ & $\begin{array}{l}\text { The number of detected } \\
\text { faults per unit time } \\
\text { increases, but the } \\
\text { uncertainty decrea- } \\
\text { ses over time. } \\
\text { (Model 3-3) }\end{array}$ \\
\hline
\end{tabular}


almost never long enough. GSRM can describe the uncertainty of applied development and calculate the uncertainty of fault detection.

We describe the uncertainty as $\sigma(t) d w(t)$, which is basically Gaussian white noise obtained from past data. The uncertainty is difficult to calculate using Eq. (5), so we assume some limits and obtain $\sigma(t) d w(t)$ quantitatively. We start by defining $a(t)$ in terms of

$$
a(t)=\alpha(t)+\sigma(t) d w(t) .
$$

Equation (5) cannot be solved due to the time dependence of $a$, as shown in Eq. (7). Therefore, we assume that $a$ is time independent with an added term $\delta$, which is small. This assumption allows Eq. (5) to be solved. These three uncertainty types can be rewritten as

$$
\begin{aligned}
& d N(t)=(\alpha(t)+\beta N(t)) N(t) d t+N(t) \delta d w(t) \\
& d N(t)=(\alpha(t)+\beta N(t)) N(t) d t+\delta d w(t) \\
& d N(t)=(\alpha(t)+\beta N(t)) N(t) d t+\frac{1}{N(t)} \delta d w(t)
\end{aligned}
$$

Each GSRM model is derived from one of these three types of uncertainty.

\subsection{Simulations}

Using these equations for GSRM, we simulated these nine cases. Figure 2 models and plots these nine cases. For each column in Table 1, the difference between each model is the parameter $\alpha(t)$. In Model 1-1, Model 2-1 and Model 3-1, which are based on Model 1-1, $a_{2}=a_{1}, a_{3}=2 a_{1}$ and $t_{1}=t_{\text {max }} / 2$ in Model 2-1, and $\alpha_{3}(t)=a_{1} t$ in Model 3-1. $\alpha(t)$ 's are set in the same manner along all columns (i.e. $\alpha(t)$ is the same along each row in Table 1). For Model 1-1, Model 2-1 and Model 3-1, the effect of uncertainty over time, $\gamma(t)=N(t) \sigma d w(t)$ increases. The situation in Model 2-1 corresponds to the number of development team members doubling at time $t 1$. The situation corresponding to Model 3-1 is that the members' skills improvement over time, effectively doubling the manpower by the time $t_{\max }$. For Model 1-2, Model 2-2, and Model 3-2, the effect of uncertainty $\gamma(t)=\sigma d w(t)$ is constant. For Model 1-3, Model 2-3 and Model 3-3, the effect of uncertainty $\gamma(t)=\sigma d w(t) / N(t)$ decreases over time.

The purpose of the simulations is to confirm that our approach can assess software reliability under dynamic changes and uncertainties in development as well as adapt the models to produce appropriate results. We used a Monte Carlo method to examine these models. Figure 2 shows the effects of uncertainties and dynamics.

\subsection{Formulation}

In Secs. 3.2 and 3.3, we simulated several stochastic differential equations. The results show that random factors affect the number of predicted faults. We assumed 

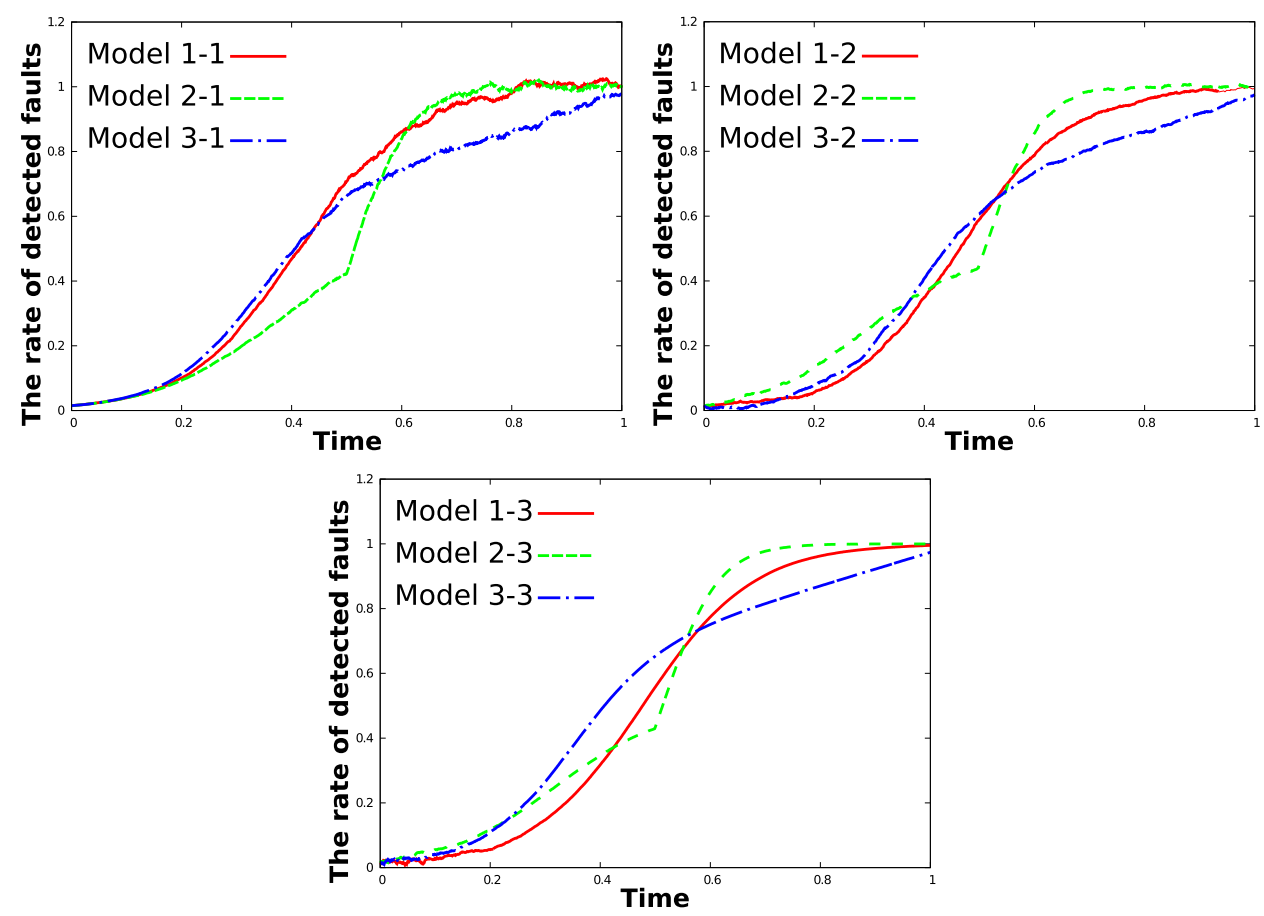

Fig. 2. Ratio of the cumulative number of detected faults at time $t$ versus the total number of detected faults for the entire project where the $x$-axis represents time in arbitrary units. 1 corresponds to $t_{\max }$ and $0: 5$ to $t_{1}$. In Model 1-1, Model 1-2 and Model 1-3, the number of detected faults per unit time is constant. In Model 2-1, Model 2-2 and Model 2-3, the number of detected faults per unit time changes at $t_{1}$. In Model 3-1, Model 3-2 and Model 3-3, the number of detected faults per unit time increases.

that the effects of random factors obey Gaussian white noise, which follows the dispersion of Eq. (6). Thus, the number of detected faults has an upper and a lower limit. In this section, we discuss the three types of uncertainty (late uncertainty, constant uncertainty, and early uncertainty) and formulate the stochastic differential equations to approximate differential equations without stochastic variables in order to treat these stochastic differential equations easily and predict the number of faults in several ranges. These approximated differential equations have simple solutions, which contain uncertainty values and will give the upper and lower limits.

\subsubsection{Late uncertainty type}

The late uncertainty type means that the uncertainties in the development circumstances increase as the development progresses. Consider the following equation:

$$
d N(t)=(\alpha+\beta N(t)) N(t) d t+N(t) \delta d w(t)
$$


If $\delta d w(t)$ is a constant value (i.e. time independent), Eq. (8) can be written as

$$
d N(t)=(\alpha+\delta+b N(t)) N(t) d t .
$$

The solution to Eq. (11) is given by

$$
N(t)=\frac{N_{\max }}{1+b \exp \{-(\alpha+\delta) t\}} .
$$

This equation is a logistic equation, where $\delta$ is the origin of the uncertainty. $\alpha+\delta$ is the gradient. The sign of $\delta$ can be positive or negative. If $\delta$ is negative (positive), the gradient of the equation is small (large). The sign of $\delta$ gives the limitation of the uncertainty. If $\delta$ is negative (positive), the growth of the graph provides a lower (upper) limit. We calculate the upper and lower limits of some projects and plot them in the next section. $\delta$ is determined as

$$
\delta_{i}=-\frac{1}{t_{i}} \ln \left\{\frac{1}{b}\left(\frac{N_{\max }}{N_{i}}-1\right)\right\}-\alpha .
$$

The subscript $i$ indicates the data is for the $i$ th fault detected at $t_{i} . i$ differs from the approximate value at $t_{i}$. Finally, we obtain the average and variance of $\delta$. We can construct the equation of SRGM from the average and variance of $\delta$ to simulate the projects and to predict when they will end by using $\delta$ and its distribution, which is Gaussian white noise [11]. We assume that the detected faults obey Eq. (12) and that the detection rate has a time-independent uncertainty $\delta$. This assumption yields the following upper and lower limits

$$
\begin{aligned}
& N_{+}(t)=\frac{N_{\max }}{1+b \exp \{-(\alpha+\delta) t\}}, \\
& N_{-}(t)=\frac{N_{\max }}{1+b \exp \{-(\alpha-\delta) t\}},
\end{aligned}
$$

where $N_{+}(t)$ means the upper limit about $N(t)$. If the development proceeds via a favorable situation, the number of detected faults will obey Eq. (14). $N_{-}(t)$ denotes the lower limit about $N(t)$. If the development proceeds via an unfavorable situation, the number of detected faults will obey Eq. (15).

\subsubsection{Constant uncertainty type}

In this section, the constant uncertainty type where the development circumstances have the uncertainties that are independent of time is discussed. Consider the following equation

$$
d N(t)=(\alpha+\beta N(t)) N(t) d t+\delta d w(t) .
$$

If $\delta \rightarrow 0$, Eq. (9) is derived as follows

$$
d N(t)=(\alpha+\beta N(t)) N(t) d t .
$$


This equation can be solved as

$$
N(t)=\frac{N_{\max }}{1+b \exp (-\alpha t)} .
$$

This is the base equation for the other types. However, if $\delta<<1$ and $\delta \neq 0$, we should consider the $\delta$ term. The entire term, which is related with $\delta$, is $\delta d w$. Because $d w$ is Gaussian white noise, the value of $\delta$ can be calculated from the actual data and base Eq. (17). We write the number of the actual detected faults as $N_{i}$ and the number of predicted faults as $N(t) . d_{i}$, which is the difference between the actual and predicted data, is defined using these two terms as

$$
d_{i}=N_{i}-N\left(t_{i}\right)
$$

$\delta$ is expressed as

$$
\delta=\sqrt{\frac{1}{m} \sum_{i=1}^{m}\left(d_{i}-\bar{d}\right)^{2}}
$$

where $\delta$ means the standard deviation. $\bar{d}$ is the average of $d_{i} . m$ represents the total number of detected faults. Finally, the upper limits and lower limits of the constant uncertainty type equations are expressed as

$$
\begin{aligned}
& N_{+}(t)=\frac{N_{\max }}{1+b \exp (-\alpha t)}+\delta, \\
& N_{-}(t)=\frac{N_{\max }}{1+b \exp (-\alpha t)}-\delta,
\end{aligned}
$$

where $N_{+}(t)$ is the upper limits about $N(t)$, while $N_{-}(t)$ means the lower limits about $N(t)$. These limit equations mean that when the number of detected faults becomes large, they are not affected by the $\delta$ uncertainty term.

\subsubsection{Early uncertainty type}

In this section, we discuss the early uncertainty type, which means that the uncertainties in the development circumstances decrease in the late stage of the development. Consider Eq. (10), which is given by

$$
d N(t)=(\alpha(t)+\beta N(t)) N(t) d t+\delta \frac{1}{N(t)} d w(t) .
$$

If $\delta \rightarrow 0$, then it can be rewritten as

$$
d N(t)=(\alpha+\beta N(t)) N(t) d t .
$$

This equation can be solved as

$$
N=\frac{N_{\max }}{1+b \exp (-\alpha t)} .
$$


This is the base equation of the other types. However, if $\delta<<1$ and $\delta \neq 0$, then this should be regarded as the $\delta$ term. The whole of the uncertainty term is $\delta d w(t) / N(t)$. Because $d w(t)$ is Gaussian white noise, the value of $\delta d w(t) / N(t)$ can be calculated from the actual data and base Eq. (17). The concrete equation is obtained from Eq. (17) as

$$
\frac{\delta\{1+b \exp (-\alpha t)\}}{N_{\max }} .
$$

$N_{i}$ is the number of actual faults and $N(t)$ is the number of predicted faults. $d_{i}$, which is the difference between the actual data and predicted data, can be expressed using these two terms as

$$
d_{i}=N_{i}-N\left(t_{i}\right)
$$

Equation (24) shows the difference between the actual data and the predicted data. Using this equation and the relation as $\delta d w / N(t), \delta$ is expressed as

$$
\delta=\sqrt{\frac{1}{m} \sum_{i=1}^{m}\left\{N\left(t_{i}\right) d_{i}\right\}^{2}} .
$$

This value $\delta$ denotes the standard deviation. Additionally, $d w$ means Gaussian white noise. Finally the equation for the early type uncertainty is written by adding Eq. (23) to Eq. (17):

$$
\begin{aligned}
& N_{+}(t)=\frac{N_{\max }}{1+b \exp (-\alpha t)}+\delta \frac{1+b \exp (-\alpha t)}{N_{\max }}, \\
& N_{-}(t)=\frac{N_{\max }}{1+b \exp (-\alpha t)}-\delta \frac{1+b \exp (-\alpha t)}{N_{\max }},
\end{aligned}
$$

where $N_{+}(t)$ means the upper limits about $N(t)$ and $N_{-}(t)$ means the lower limits about $N(t)$. These equations indicate that when the number of detected faults is large, the uncertainty term has a negligible influence on the number of detected faults.

\section{Evaluation}

In this section, we apply the three types of uncertainties to four projects from two datasets to determine which uncertainty types are suitable for each dataset. The first development dataset is from [8]. The second development dataset is from [29]. Moreover, we compared GSRM with other SRGMs using two datasets.

\subsection{Evaluation design and results}

We evaluate GSRM by applying to two datasets [8, 29] and comparing our model with other models. First, we applied the three uncertainty types of GSRM to these 
datasets to determine which uncertainty type is suitable for the development situation. To analyze the suitability, we evaluated the coverages between their upper and lower limits. The areas between these limits contain several actual data points. If the number of contained actual data points of one type is larger than that of the other type, we considered the uncertainty type is suitable for the dataset. Second, we compared GSRM with other models with respect to the accuracy of applying the models to these datasets. We adopted the residual sum of square (RSS) and Akaike Information Criteria (AIC). RSS means the differences between the model and the data. AIC means the differences between the model and the data considering the number of parameters in the model. If the RSS and AIC values are small, the model fits with the data. Because these values indicate model fitness, the fitness of different models can be compared. Our evaluation uses the datasets in Table 2. DS 1 [8] was obtained in 1979. DS 2-1, 2-2, and 2-3 [29] were obtained in 2000. DS 2-1, 2-2, and 2-3 were developed with several testing guidelines, including the fault-prone method, and using previous projects' data [30].

Table 2. Details of each dataset.

\begin{tabular}{cccccc}
\hline Dataset & Year & Term & Faults & Citation & Explain \\
\hline DS 1 & 1979 & 849 days & 34 & {$[8]$} & US Navy \\
DS 2-1 & 2000 & 18 weeks & 176 & {$[29]$} & Pharmacy (release 1) \\
DS 2-2 & 2000 & 17 weeks & 204 & {$[29]$} & Pharmacy (release 2) \\
DS 2-3 & 2000 & 13 weeks & 77 & {$[29]$} & Pharmacy (release 3) \\
\hline
\end{tabular}

\subsubsection{Design}

This development dataset is from [8] and is written by Goel and Okukmoto. The data are originally from the U.S. Navy Fleet Computer Programming Center and consist of the errors in software development (Table 3). The second development dataset is from [29] and is written by Stringfellow et al. The data come from three releases of a large medical record system, which consists of 188 software components (Table 4). The data contain the cumulative number of faults and their detected times for the three different releases of the software program. We applied GSRM to these datasets and classified the datasets based on the uncertainty type by comparing the covered actual data points between the upper and lower limits. To compare with NHPP

Table 3. Dataset 1 (DS 1). Each row contains the total number of faults detected over the corresponding number of days.

\begin{tabular}{crrrrrrrrrrrrr}
\hline Error no. & 1 & \multicolumn{1}{c}{2} & \multicolumn{1}{c}{3} & \multicolumn{1}{c}{4} & \multicolumn{1}{c}{5} & \multicolumn{1}{c}{6} & \multicolumn{1}{c}{7} & 8 & 9 & 10 & 11 & 12 & 13 \\
\hline Time (days) & 9 & 21 & 32 & 36 & 43 & 45 & 50 & 58 & 63 & 70 & 71 & 77 & 78 \\
Error no. & 14 & 15 & 16 & 17 & 18 & 19 & 20 & 21 & 22 & 23 & 24 & 25 & 26 \\
Time (days) & 87 & 91 & 92 & 95 & 98 & 104 & 105 & 116 & 149 & 156 & 247 & 249 & 250 \\
Error no. & 27 & 28 & 29 & 30 & 31 & 32 & 33 & 34 & - & - & - & - & - \\
Time (days) & 337 & 384 & 396 & 405 & 540 & 798 & 814 & 849 & - & - & - & - & - \\
\hline
\end{tabular}


Table 4. Dataset 2 (DS 2-1, 2-2, 2-3). Each row contains the total number of faults detected over the corresponding number of weeks.

\begin{tabular}{lccc}
\hline Weeks & Release 1 (DS 2-1) & Release 2 (DS 2-2) & Release 3 (DS 2-3) \\
\hline 1 & 28 & 90 & 9 \\
2 & 29 & 107 & 14 \\
3 & 29 & 126 & 21 \\
4 & 29 & 145 & 28 \\
5 & 29 & 171 & 53 \\
6 & 37 & 188 & 56 \\
7 & 63 & 189 & 58 \\
8 & 92 & 190 & 63 \\
9 & 116 & 190 & 70 \\
10 & 125 & 190 & 75 \\
11 & 139 & 192 & 76 \\
12 & 152 & 192 & 77 \\
13 & 164 & 192 & - \\
14 & 164 & 192 & - \\
15 & 165 & 203 & - \\
16 & 168 & 203 & - \\
17 & 170 & 204 & - \\
18 & 176 & - & \\
\hline
\end{tabular}

models (NHPP and S-shaped), we adopted the time independent model of GSRM because these datasets do not have person month data. The models were evaluated using RSS and AIC where small values indicate that the model has a sufficient fit.

Table 2 shows that these datasets contain data from the 1970s and the 2000s, which were produced by waterfall developments. The 2000s projects were developed with the fault prone method, which is a modern approach [30]. Therefore, the 2000s projects were developed via more modern processes than the 1970 s project.

\subsubsection{Results}

We evaluated the adoptions for the three uncertainty types and compared the GSRMs with NHPP models through the two datasets. For each uncertainty type, we calculated the upper limit and the lower limit as well as its suitability. Figures 3-6 and Table 5 show the results. We compared the GSRMs with the NHPP models (e.g. the normal NHPP model and S-shaped model) through RSS and AIC. As mentioned above, small RSS and AIC values indicate a good model fitness. Figure 7 and Table 6 show the results.

\subsubsection{Type selection}

We calculated $\delta$ and the upper and lower limits. Figures 3-6 plot the results where the $x$-axis represents time and the $y$-axis represents the number of detected faults. Solid, dashed, and dotted-dashed lines represent values calculated with GSRM, upper limits, and lower limits, respectively. The crosses indicate the actual data in [8] and [29]. These results confirm that almost all of the real data points are contained 


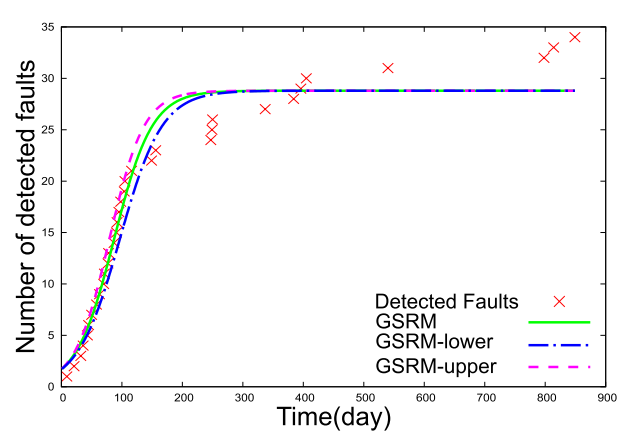

(a)

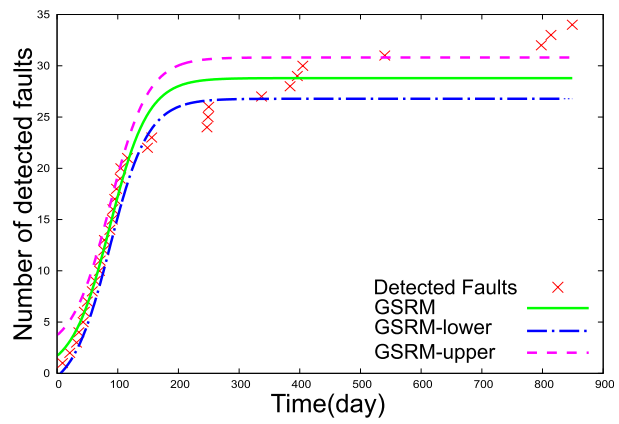

(b)

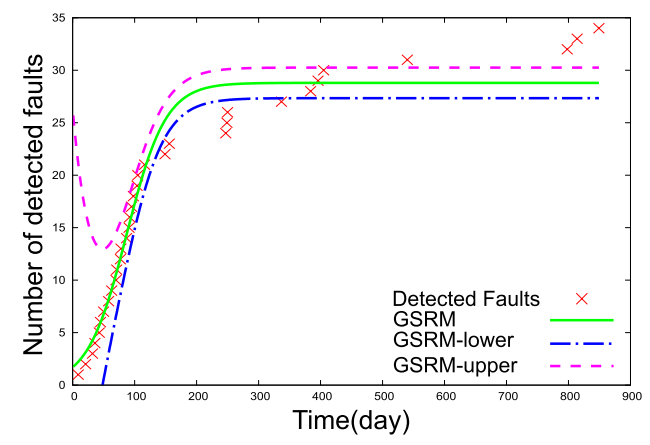

(c)

Fig. 3. Cumulative number of detected faults for the entire project of DS 1 plotted against the elapsed number of days. In the legend, Detected faults, GSRM, GSRM-upper, and GSRM-lower represent the actual data, the fit using GSRM, the predicted upper limit, and the predicted lower limit, respectively. (a) The late uncertainty type, (b) the constant uncertainty type, and (c) the early uncertainty type.

within the calculated upper and lower limits. We quantitatively evaluated which type is suitable for each datasets by calculating the Coverage, Area, and Rate for each dataset. Coverage means the number of actual data points between the upper and lower limits. Area, which denotes the area surrounded by the upper and lower models, is calculated by integrating the upper and lower models. Rate, which indicates the coverage rate of the type, represents the Coverage divided by Area. Coverage is the most important value since a model with a large Coverage can cover a lot of actual data points. If one type has a larger Coverage than the other types, it is more suitable for the dataset. However, if the types have equal Coverage, then the type with the largest Rate is most suitable for the dataset.

Table 5 shows the results. The constant type of uncertainty is suitable for dataset 1 because it has the largest coverage (Fig. 3 and Table 6). This is reasonable since the interval of detecting faults is random as the development proceeds in dataset 1 , indicating that uncertainty events randomly occur. The results of release 1,2 , and 3 indicate that the late type is suitable for dataset 2 because it has the largest coverage 


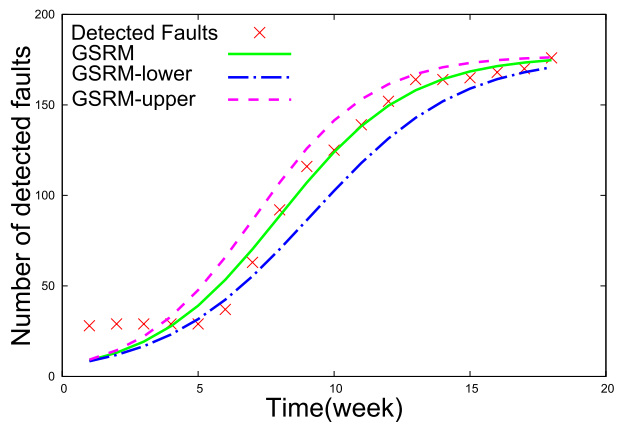

(a)

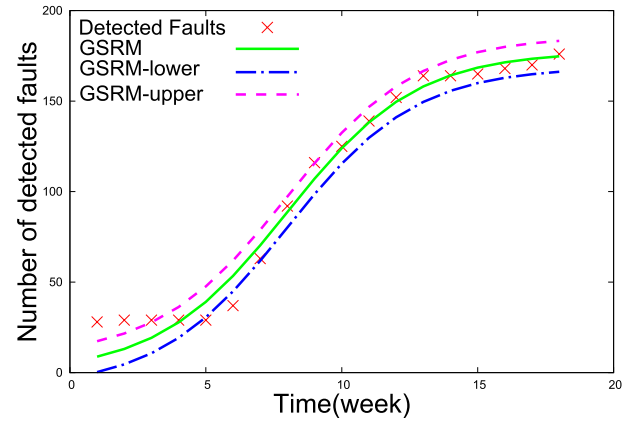

(b)

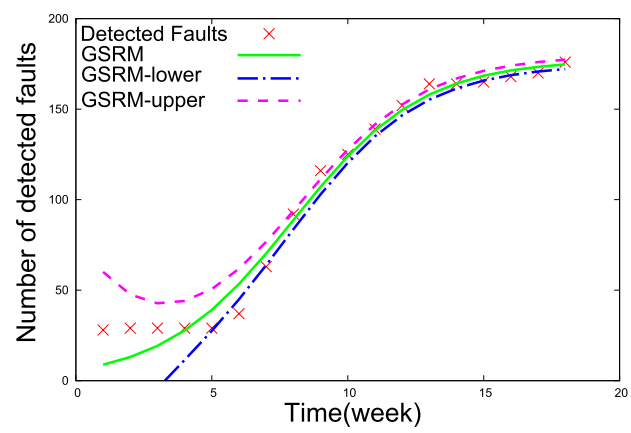

(c)

Fig. 4. Cumulative number of detected faults for the entire project of DS 2-1 plotted against the elapsed number of weeks. Legends and the titles are the same as Fig. 3.

(Figs. 4-6). This is consistent with Tables 3 and 6 where more faults are detected at the end of development for dataset 2, indicating that the development has issues up until each release.

In these datasets, we could not verify the results by interviewing the development teams that produced these datasets due to the age of the datasets. Therefore, we requested that a Japanese IT company, which employs about 5000 people, use GSRMs and evaluate the results, including the uncertainties, from 2013 to 2015 . We collected the fault datasets from the two projects in the company. Additionally, the two managers of the two development teams evaluated the results. Then we asked the two managers about the GSRM results and whether they agreed with the uncertainty type according to the GSRMs. They responded that the uncertainty type indicated by the results is consistent with their thoughts.

\subsubsection{Comparison}

The NHPP models are well-known reliability models. In this section, we discuss the differences between GSRM and NHPP models using the actual development data in 


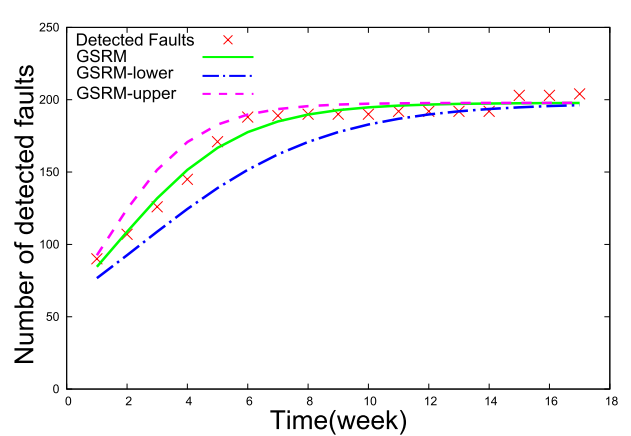

(a)

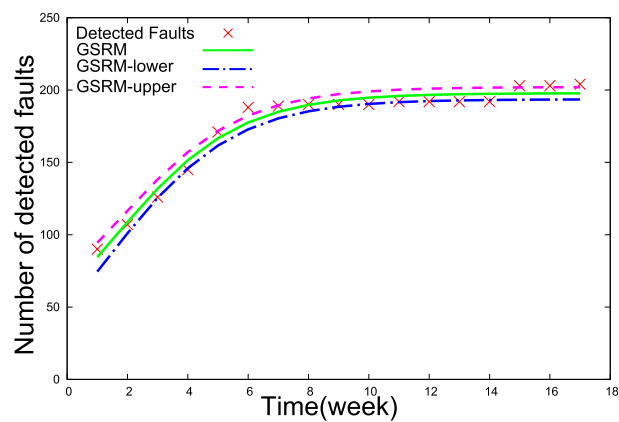

(b)

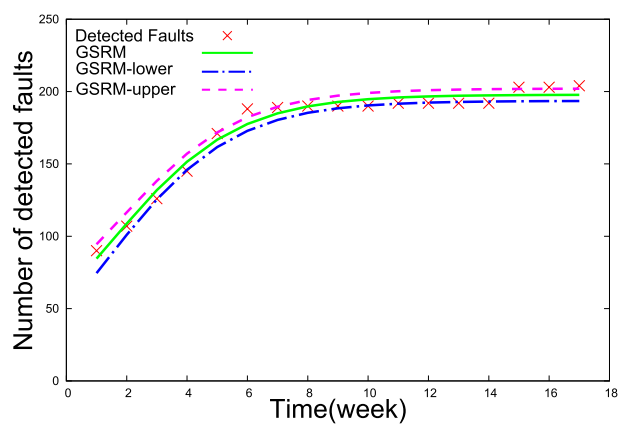

(c)

Fig. 5. Cumulative number of detected faults for the entire project of DS 2-2 plotted against the elapsed number of weeks. Legends and the titles are the same as Fig. 3.

a given situation when the growth rate is time independent. The reason for this limitation is because the NHPP model cannot be applied to time-dependent situations. Figure 7 graphs the results, where the crosses represent the actual data in [8] and [29]. The solid, dashed, and dotted-dashed lines represent the GSRM fitted to the actual data, the NHPP fitted to the actual data, and the S-shaped model fitted to the actual data, respectively. The parameters are calculated by $\mathrm{R}$ [1], which is a language and environment for statistical computing and graphics. DS 1 and DS 2-2 are the same projects as shown Fig. 1, which is the motivating example. To verify that GSRM is a better model than the other models, we calculate the RSSs and the AICs from these models and the development data (Table 6).

The RSSs and AICs of the GSRM are lower in DS 2-1, DS 2-2 and DS 2-3 than those of the NHPP and S-shaped models, suggesting that GSRM is a better approximation than the NHPP models because GSRM has more flexibility due to the nonlinear term. Especially, the GSRM has the breaking term such as $\beta$, which causes the model to converge on the given values near the actual data points. 


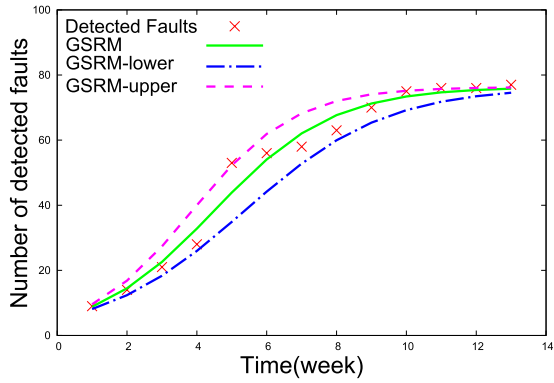

(a)

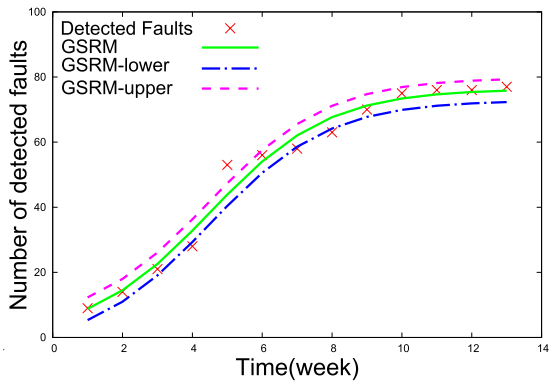

(b)

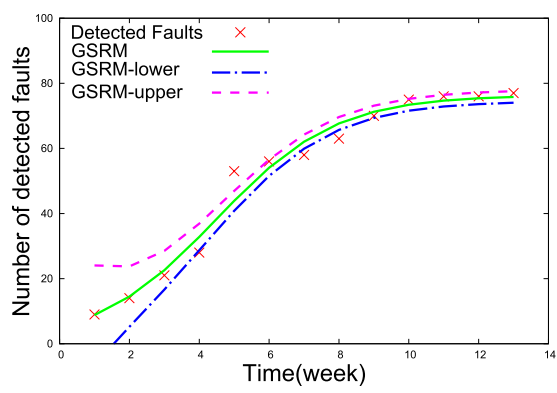

(c)

Fig. 6. Cumulative number of detected faults for the entire project of DS 2-3 plotted against the elapsed number of weeks. Legends and titles are the same as Fig. 3.

Table 5. Selection of three uncertainty types for datasets 1 and 2 .

\begin{tabular}{|c|c|c|c|c|c|c|c|c|c|}
\hline & \multicolumn{3}{|c|}{ Late type } & \multicolumn{3}{|c|}{ Constant type } & \multicolumn{3}{|c|}{ Early type } \\
\hline & Coverage & Area & Rate & Coverage & Area & Rate & Coverage & Area & Rate \\
\hline DS 1 & 16 & 62.9 & 0.254 & 25 & 136.9 & 0.183 & 24 & 271.3 & 0.088 \\
\hline DS 2-1 & 13 & 350.6 & 0.037 & 12 & 307.1 & 0.039 & 11 & 374.9 & 0.029 \\
\hline DS 2-2 & 13 & 343.5 & 0.038 & 9 & 179.5 & 0.050 & 8 & 174.3 & 0.046 \\
\hline DS 2-3 & 10 & 114.3 & 0.087 & 9 & 90.9 & 0.099 & 9 & 106.6 & 0.084 \\
\hline
\end{tabular}

Table 6. Comparison of GSRM with the NHPP models using datasets 1 and 2 .

\begin{tabular}{ccccc}
\hline & & NHPP & S-Shaped & GSRM \\
\hline DS 1 & RSS & 131.3 & 100.1 & 137.8 \\
& AIC & 148.4 & 139.2 & 152.1 \\
DS 2-1 & RSS & 4612 & 3246 & 1310 \\
& AIC & 158.9 & 152.6 & 136.3 \\
DS 2-2 & RSS & 696.1 & 3489 & 473.7 \\
& AIC & 119.4 & 145.8 & 112.8 \\
DS 2-3 & RSS & 264.8 & 181.1 & 158.8 \\
& AIC & 84.07 & 79.14 & 77.43 \\
\hline
\end{tabular}



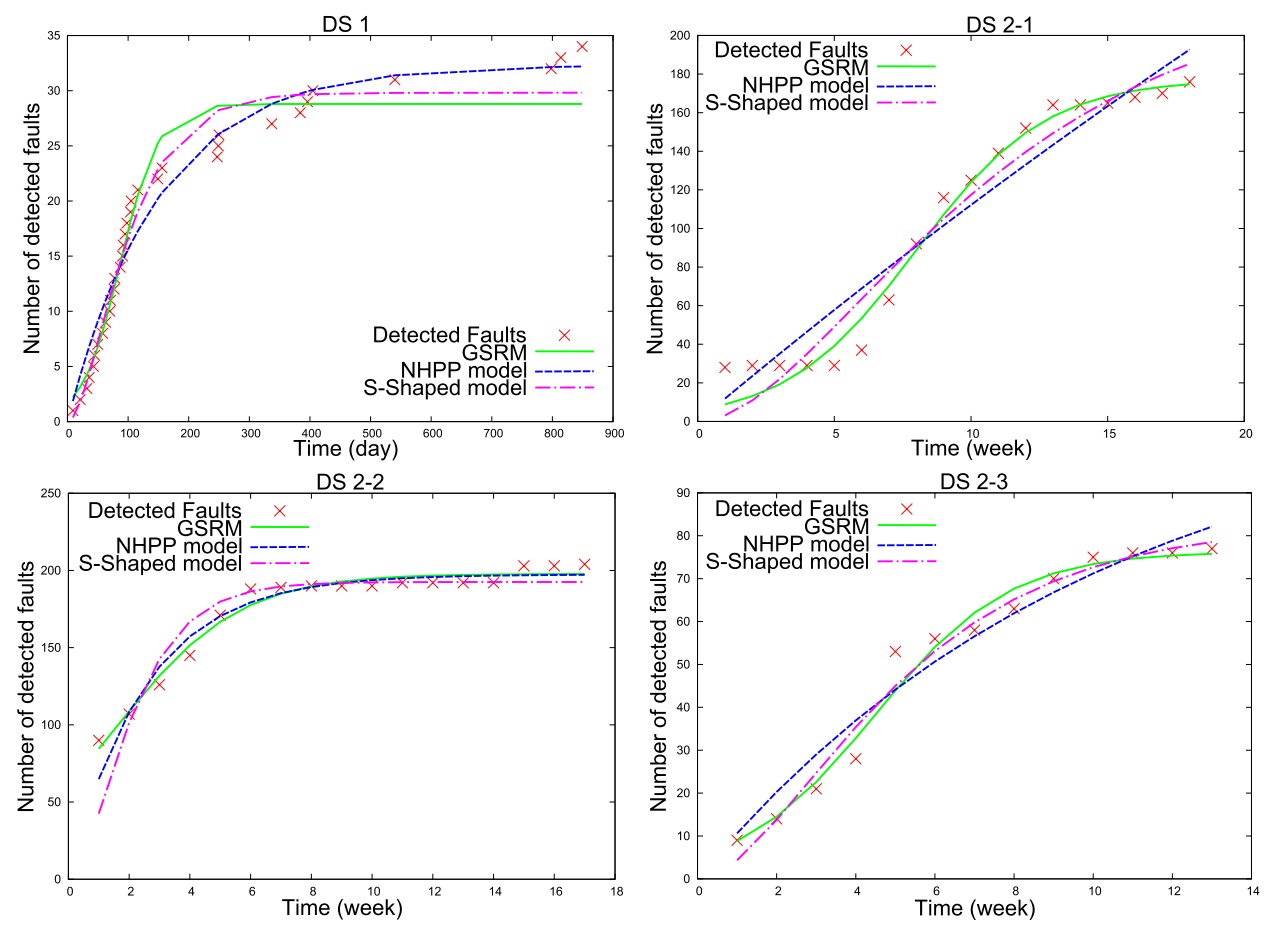

Fig. 7. In "DS 1", the cumulative number of detected faults for DS 1 is plotted against the elapsed number of days. In the legend, Detected faults, GSRM, NHPP model, and S-shaped model represent the actual data, the fit using GSRM, the fit using the NHPP model, and the S-shaped NHPP model, respectively. In the other graphs, the cumulative number of detected faults for 1 project of dataset 2 is plotted against the elapsed number of weeks. Legends are the same as "DS 1."

\subsection{Discussion}

We compared GSRM with other NHPP models using two datasets in order to assess whether GSRM can describe the data precisely. Additionally, we applied the three uncertainty types to the datasets to verify whether GSRM can adapt to different situations.

\subsubsection{Wide applicability (RQ1)}

In our simulations, we applied the reliability growth models to nine types of development situations, which are characterized by two uncertainty elements related to the detection of faults.

We successfully simulated the three types of dynamics of the development situation: the number of detected faults per unit time is constant (e.g. the number of members is constant), the number of detected faults per unit time changes at the given time (e.g. new members join the project a given time) and the number of detected faults per unit time increases (e.g. new members join the project gradually). 
Especially, for the type where the number of detected faults per unit time increases, the number of detected faults is smaller than the other types in the late development, but it is greater than the other types in early development. This means that if new members join the project gradually, the number of detected faults will be smaller than other types at the late of the development. If managers decided to increase the members at the early development, ideally they would add new members all at once and not gradually.

Existing models can describe only one of these situations with additional limitations, but GSRM can describe several of these situations primarily because existing models cannot handle time-dependent growth rates without limitations, whereas GSRM can handle time-dependent rates as long as the appropriate uncertainty situation is inputted. Additionally, GSRM has a scheme for development uncertainties and can construct a model involving uncertainties.

GSRM has a good fit for almost all datasets. The S-shape model has a good fit for several datasets, but a worse fit for other datasets (e.g. dataset 2, release 2), indicating that the appropriateness of the model depends on the situation. GSRM shows that dataset 2 , release 2 is the late uncertainty type, while the other releases have similar coverages for the different uncertainty types. The results suggest that dataset 2 , release 2 may be complicated since many faults are detected in the first week. Regardless, these results indicate that GSRM can treat several situations.

The old dataset (e.g. dataset 1) is a constant type of GSRM, while the new datasets (e.g. dataset 2) are late types of GSRM. This means that the old development has uncertainty events throughout development, while the new development has uncertainty events at the later stage of development. Nowadays, many researchers and developers recommend that faults or bugs be removed at the earlier stages of development. We assume that the new development would be well controlled since modern developers may have more skills and knowledge than traditional developers. On the other hand, we assume that the old development would be always exposed to uncertainty events since developers may not have sophisticated skills and specific knowledge.

\subsubsection{Comparison with other models (RQ2)}

Given a situation where the growth rate is time independent, we used two actual datasets to compare to GSRM with the NHPP models. The results show a highprecision convergence of the numbers of faults and appropriate development terms with GSRM. The precision of convergence is at least $12 \%$ higher for GSRM than for the NHPP models, confirming that GSRM can describe software growth more realistically than previously proposed models based on the NHPP models. Thus, using GSRM may help developers devise a more accurate plan for releasing software. 


\subsection{Limitations}

We derive three uncertainty types by considering development situations artificially. However, the three types are intuitive.

To verify the probability of the types (late type, constant type, and early type), we interviewed several developers about whether these three types are suitable for actual developments. Almost all developers responded affirmatively and answered that their experiments seem to correspond to these types. Although one developer answered that there are other uncertainty types, the framework that the product employed in his experiment was forced to suddenly change to another framework mid-development. Although we propose three types of uncertainties in development as an initial study, it is true that there are other types of uncertainties.

\subsubsection{Threats to internal validity}

We treated the growth of the number of faults as a time-dependent function. The growth of the number of faults may be related to other factors (e.g. test efforts). In this research, we could not collect and evaluate the test efforts and other data. The test efforts should influence the number of the detected faults because if the test efforts are not constant, the number of detected faults changes.

We chose three types of uncertainty: late, constant, and early. It is not necessary to divide the uncertainty types by time categories. Although the responses vary by individual, we asked several developers whether their assessment agreed with the uncertainty type of the GSRM. Overall, the developers' thoughts were consistent with the uncertainty types.

In comparing the models, we used two datasets, both of which were obtained by one organization or company. It is possible that the data contains mistakes or other false elements. Several studies have focused on the accuracy of the faults datasets. An early paper on bug reports in open software indicates that the bug reports often contain information that does not describe bugs [10]. However, the datasets we used were collected from industries. In general, industries try to collect exact bug reports because they do not want to release software with bugs. If bugs remain in software released to other companies, the bugs may have a detrimental effect. Thus, products developed by industries tend to contain few faults. In [29], products related to dataset 2 have few faults in each release. Hence, the number of mistakes remaining in the dataset does not greatly impact our research results.

Additionally, the data were too old to compare with recent developments. However, recent studies have also employed these datasets, which should protect the validity of the results of this study.

\subsubsection{Threats to external validity}

We only tested GSRM with two datasets, which is insufficient to make generalizations about GSRM. Moreover, the datasets are old and the scales of their systems 
are smaller than recent systems. Although a lot of factors (e.g. development styles, development scales, organizations, ages, etc.) should greatly affect the growth of the number of faults, we could not evaluate such factors. We evaluated two datasets belonging to a different organization published in different timeframes.

In the future, we plan to use datasets related to large-scale systems. Moreover, we plan to use datasets with different development styles or development scales. Additionally, we only compared GSRM with NHPP models. However, other models exist. Although these models have similar origins as the NHPP model, GSRM should be compared to other models besides NHPP models.

\subsubsection{Threats to construct validity}

We supposed that the uncertainty types of developments can be categorized as late type, constant type, and early type, and can be evaluated by applying GSRM to actual datasets. The types of uncertainties were artificial and may not be applicable to actual datasets because it is possible that the datasets belong to other uncertainty types.

Additionally, it is unclear whether we evaluated the correct uncertainty values by applying GSRMs to the datasets quantitatively since our definitions of the uncertainty values were built from only the viewpoints of the number of faults and time series. However, several developers who understood the results of GSRM concurred with the types determined by GSRM.

\section{Related Works}

\subsection{SRGMs}

Many kinds of SRGMs exist. Several researchers proposed time-dependent models whose situations are limited such as the error detection per time increase with the progress of software testing [39]. Yamada et al. proposed an extend NHPP model, which is related with test-domain dependence [40]. The test-domain dependent model includes the idea that the tester's skills should improve by degrees (e.g. the growth of skills is time dependent). Hou et al. proposed a SRGM considering two learning curves, which are the exponential learning curve and the S-shaped learning curve [17]. These models assume that the number of developers does not change and the time-dependent parameters are model specific like the exponential learning curve. Our model does not depend on such specific models and situations.

Although software reliability models have not been used on waterfall development, Fujii et al. developed a quantitative software reliability assessment method in incremental development processes, which is an agile software development, based on the familiar non-homogeneous Poisson processes [6]. They used not only the number of faults but also software metrics, which are the number of software modules, the number of design reviews, the number of test cases performed, the size of software, and the development effort. They showed a software reliability 
prediction through a case study. They developed a SRGM for the incremental development processes and adopted other metrics to their SRGM, but they did not directly treat the number of developers. Our model can directly treat the number of developers, which should be suitable to actual and recent development styles.

Kuo et al. proposed a framework for modeling the software reliability model using various testing efforts and fault detection rates [21]. The testing efforts mean the resource expenditures spent on software testing (e.g. test cases, human resource, CPU time) applied into SRGMs in [36]. Kuo et al. applied their framework to several NHPP models by employing three types of testing effort functions (constant testing effort consumption, Weibull-type testing effort function, and Logistic testing effort functions) and two types of fault detection rates (constant proportionality and timevariable fault detection rates).

Additionally, Ahmad et al. proposed an S-shaped NHPP model containing testing-effort [2]. Ahmad et al. assumed that the testing effort expenditures are described by the Log-logistic function and integrated the Log-logistic function into an S-shaped NHPP model. Their model requires the test effort expenditures such as the test cases and human resource, but our model only needs the number of developers.

Huang et al. proposed five SRGMs with multiple changing points, which could be treated as the timing of introducing new tools or techniques [18]. They prepared datasets with and without multiple changing points, and evaluated their SRGMs with the changing points and the estimated changing points. Their idea about multiple changing points in development is similar to our assumption about dynamic changes in development. However, they only target the timing of the changes of development, whereas our model targets the timing and the concrete values such as the increased numbers of developers. Since our model can treat concrete values such as the numbers developers, we are able to simulate the development in Sec. 3.3.

Singh et al. proposed a SRGM using a feed forward neural network approach, which is a machine learning method [28]. Their approach uses part of the data $(60 \%$ to $80 \%$ from the beginning) for several datasets to train each neural network model. We supposed that if several development changes occur after learning data their model would not be able to treat such situations since it was constructed with the learning data using a machine learning technique. Our model can treat a situation such as changes in developers, which we simulate in Sec. 3.3.

\subsection{Uncertainties}

Several researchers tried to treat the uncertainties in requirements and operations. Wallace et al. studied the risk [32] and analyzed the software project risks to reduce the incidence of failure [32]. They mentioned that software projects have six dimensions: Team Risk, Organizational Environment Risk, Requirements Risk, Planning and Control Risk, User Risk, and Complexity Risk. They emphasized that Organizational Environment Risk and Requirements Risk are due to risks and uncertainties. Our studies focus on the uncertainties and try to evaluate the 
uncertainties in a quantitative manner. In contrast, Wallace et al. did not focus on the uncertainties and did not evaluate the uncertainties in a quantitative way.

Goseva-Popstojanova and Kamavaram studied the uncertainties in requirements and operations by component-based software engineering [9, 19]. In [19], they analyzed the uncertainties of operational profiles and component reliability by calculating the conditional entropy of each component. In [9], they analyzed the uncertainties of the operational profiles and the component reliability by Monte Carlo simulations. These analytic methods focus on the requirements and operations. However, our methods focus on the uncertainties in development phases, including requirements and operations. In the future, we plan to analyze the effects of the uncertainties in requirements and operations since we suppose that the uncertainties in the requirement phases affect the development process.

\section{Conclusion}

Using GSRM, we successfully simulated developments containing uncertainties and dynamic elements. We obtained the time-dependent logistic curve and growth curve, which is not possible using other models, as well as simulated and analyzed nine types of developments with GSRM. Additionally, we formulated equations for three types of uncertainty that are related to actual development situations. We also defined uncertainty values from actual data containing information on faults during development and applied GSRM to datasets to calculate the fitness of the models. These results demonstrate that GSRM can calculate uncertainties using past data and predict how long a project will take.

In the future, we plan to evaluate teams or team members using quantitative methods while considering uncertainties to optimize teams for a particular project using GSRM.

\section{Acknowledgments}

We are grateful to Ken Asoh, Kazuyoshi Takahashi, Kentarou Ogawa, Maki Mori, Takashi Hino, Yosuke Hayakawa, Yasuyuki Tanaka, Shinichi Yamada and Daisuke Miyazaki (Yahoo Japan Corporation) for helpful discussions.

This work has been conducted as a part of "Research Initiative on Advanced Software Engineering in 2015" supported by Software Reliability Enhancement Center (SEC), Information Technology Promotion Agency Japan (IPA). This work was also supported by JSPS KAKENHI Grants Nos. 25330091 and 16H02804.

\section{References}

1. The R project for statistical computing. Available at http://www.r-project.org/.

2. N. Ahmad, M. G. Khan and L. S. Rafi, Analysis of an inflection s-shaped software reliability model considering log-logistic testing-effort and imperfect debugging. Int. J. Comput. Sci. Netw. Secur. 11(1) (2011) 161-171. 
3. M. Anjum, M. A. Haque and N. Ahmad, Analysis and ranking of software reliability models based on weighted criteria value, Int. J. Inform. Tech. Comp. Sci. 5(2) (2013) 1.

4. X. Cai and M. Lyu, Software reliability modeling with test coverage: Experimentation and measurement with a fault-tolerant software project, in 18th IEEE Int. Symp. Software Reliability, 2007, pp. 17-26.

5. T. Dohi, K. Yasui and S. Osaki, Software reliability assessment models based on cumulative bernoulli trial processes, Mathematical and Computer Modelling 38(11) (2003) $1177-1184$.

6. T. Fujii, T. Dohi and T. Fujiwara, Towards quantitative software reliability assessment in incremental development processes, in Proc. 33rd Int. Conf. Software Engineering, 2011, pp. 41-50.

7. A. Goel, Software reliability models: Assumptions, limitations, and applicability, Softw. Eng. IEEE Trans. 11(12) (1985) 1411-1423.

8. A. L. Goel and K. Okumoto, Time-dependent error-detection rate model for software reliability and other performance measures, IEEE Trans. Reliab. 3 (1979) 206-211.

9. K. Goseva-Popstojanova and S. Kamavaram, Assessing uncertainty in reliability of component-based software systems, in 14 th Int. Symp. Software Reliability Engineering, 2003, pp. 307-320.

10. K. Herzig, S. Just and A. Zeller, It's not a bug, it's a feature: How misclassification impacts bug prediction, in Proc. Int. Conf. Software Engineering, 2013, pp. 392-401.

11. K. Honda, H. Nakai, H. Washizaki, Y. Fukazawa, K. Asoh, K. Takahashi, K. Ogawa, M. Mori, T. Hino, Y. Hayakawa et al., Predicting time range of development based on generalized software reliability model, in 21st Asia-Pacific Software Engineering Conf., 2014.

12. K. Honda, N. Nakamura, H. Washizaki and Y. Fukazawa, Case study: Project management using cross project software reliability growth model, in IEEE Int. Conf. Software Quality, Reliability and Security Companion, 2016, pp. 39-46.

13. K. Honda, N. Nakamura, H. Washizaki and Y. Fukazawa, Case study: Project management using cross project software reliability growth model considering system scale, in IEEE Int. Symp. Software Reliability Engineering Workshops, 2016.

14. K. Honda, H. Washizaki and Y. Fukazawa, A generalized software reliability model considering uncertainty and dynamics in development, in Product-Focused Software Process Improvement, eds. J. Heidrich et al., LNCS Vol. 7983, 2013, pp. 342-346.

15. K. Honda, H. Washizaki and Y. Fukazawa, Predicting release time based on generalized software reliability model (GSRM), in 38th Annual Conference on Computer Software and Applications, 2014, pp. 604-605.

16. K. Honda, H. Washizaki, Y. Fukazawa, K. Munakata, S. Morita, T. Uehara and R. Yamamoto, Detection of unexpected situations by applying software reliability growth models to test phases, in Int. Symp. Software Reliability Engineering Workshops, 2015, pp. $2-5$.

17. R.-H. Hou, S.-Y. Kuo and Y.-P. Chang, Applying various learning curves to hypergeometric distribution software reliability growth model, in Proc. 5th Int. Symp. Software Reliability Engineering, 1994, pp. 8-17.

18. C.-Y. Huang and M. R. Lyu, Estimation and analysis of some generalized multiple change-point software reliability models, Reliability, IEEE Trans. 60(2) (2011), 498-514.

19. S. Kamavaram and K. Goseva-Popstojanova, Entropy as a measure of uncertainty in software reliability, in 13th Int. Symp. Software Reliability Engineering, 2002, pp. 209-210.

20. Y. Kamei, A. Monden and K.-I. Matsumoto, Empirical evaluation of svm-based software reliability model, in Proc. Fifth ACM/IEEE Int. Symp. Empirical Software Eng. 2 (2006) $39-41$. 
21. S.-Y. Kuo, C.-Y. Huang and M. R. Lyu, Framework for modeling software reliability, using various testing-efforts and fault-detection rates, IEEE Trans. Reliab. 50(3) (2001) 310-320.

22. R. Lai and M. Garg, A detailed study of nhpp software reliability models, J. Softw. 7(6) (2012) 1296-1306.

23. J. D. Musa and K. Okumoto, A logarithmic poisson execution time model for software reliability measurement, in Proc. 7th Int. Conf. Software Engineering, 1984, pp. 230-238.

24. E. Nguyen, C. Rexach, D. Thorpe and A. Walther, The importance of data quality in software reliability modeling, in 21st Int. Symp. Software Reliability Engineering, 2010, pp. 220-228.

25. H. Okamura, Y. Etani and T. Dohi, A multi-factor software reliability model based on logistic regression, in 21st Int. Symp. Software Reliability Engineering, 2010, pp. 31-40.

26. R. Rana, M. Staron, C. Berger, J. Hansson, M. Nilsson and F. Torner, Evaluating longterm predictive power of standard reliability growth models on automotive systems, in 24th Int. Symp. Software Reliability Engineering, 2013, pp. 228-237.

27. N. Schneidewind and M. Hinchey, A complexity reliability model, in 20th Int. Symp. Software Reliability Engineering, 2009, pp. 1-10.

28. Y. Singh and P. Kumar, Prediction of software reliability using feed forward neural networks, in Int. Conf. Computational Intelligence and Software Engineering, 2010, pp. $1-5$.

29. C. Stringfellow and A. Andrews, An empirical method for selecting software reliability growth models, Emp. Softw. Eng. 7(4) (2002) 319-343.

30. C. V. Stringfellow, An integrated method for improving testing effectiveness and efficiency, 2007.

31. Y. Tamura and S. Yamada, A flexible stochastic differential equation model in distributed development environment, Eur. J. Operat. Res. 168(1) (2006) 143-152.

32. L. Wallace, M. Keil and A. Rai, Understanding software project risk: A cluster analysis, Inform. Manag. 42(1) (2004) 115-125.

33. H. Washizaki, K. Honda and Y. Fukazawa, Predicting release time for open source software based on the generalized software reliability model, in Agile Conf., 2015, pp. 76-81.

34. K. Worwa, A discrete-time software reliability-growth model and its application for predicting the number of errors encountered during program testing, Contr. Cybern. 34(2) (2005) 589.

35. S. Yamada, Recent developments in software reliability modeling and its applications, in Stochastic Reliability and Maintenance Modeling (Springer, 2013), pp. 251-284.

36. S. Yamada, J. Hishitani and S. Osaki, Software-reliability growth with a weibull testeffort: A model and application, IEEE Trans. Reliab. 42(1) (1993) 100-106.

37. S. Yamada, M. Kimura, H. Tanaka and S. Osaki, Software reliability measurement and assessment with stochastic differential equations, Trans. Fundamentals of Electronics, Communications and Computer Sciences 77(1) (1994) 109-116.

38. S. Yamada, M. Ohba and S. Osaki, S-shaped reliability growth modeling for software error detection, IEEE Trans. Reliab. 32(5) (1983) 475-484.

39. S. Yamada, M. Ohba and S. Osaki, s-shaped software reliability growth models and their applications, IEEE Trans. Reliab. 33(4) (1984) 289-292.

40. S. Yamada, H. Ohtera and M. Ohba, Testing-domain dependent software reliability models, Comput. Math. Appl. 24(12) (1992) 79-86.

41. N. Zhang, G. Cui and H. Liu, A stochastic software reliability growth model with learning and change-point, in World Automation Congress, 2012, pp. 399-403. 